REVIEW ARTICLE

P.C. Sundgren

\section{MR Spectroscopy in Radiation Injury}

\begin{abstract}
SUMMARY: Detecting a new area of contrast enhancement in or in the vicinity of a previously treated brain tumor always causes concern for both the patient and the physician. The question that immediately arises is whether this new lesion is recurrent tumor or a treatment effect. The differentiation of recurrent tumor or progressive tumor from radiation injury after radiation therapy is often a radiologic dilemma regardless the technique used, CT or MR imaging. The purpose of this article was to review the utility of one of the newer MR imaging techniques, MR spectroscopy, to distinguish recurrent tumor from radiation necrosis or radiation injury.
\end{abstract}

$\mathbf{N}$ ew contrast enhancing lesions discovered on routine follow-up brain imaging at or near the site of previously treated primary brain tumor present a diagnostic dilemma. Posttreatment imaging features are often non-specific and the differentiation between recurrent tumor and radiation injury is often difficult. In attempts by investigators to improve local tumor control and the overall clinical outcome and survival for patients with primary brain tumor, new, more aggressive treatment protocols are implemented or tested. These protocols include different schemes of dosages of various chemotherapeutic agents but also different schemes of locally administered high doses of radiation.

Although these new radiation schemes have resulted in improved outcome, they have also been associated with a significant incidence of radiation injury to the brain. It is well documented that there is a relationship between increased survival and increased total dose. ${ }^{1}$ The risk of late effects that can lead to devastating functional deficits several months to years after brain irradiation limits the total dose that can safely be administrated to patients. Recent data suggest that progressive dementia occurs in approximately $20 \%-50 \%$ of patients with brain tumor who are long-term survivors after treatment with large-field partial- or whole-brain irradiation. ${ }^{2}$

The differentiation of recurrent tumor or progressive tumor from radiation injury after radiation therapy is often a radiologic dilemma, regardless of the technique used, CT or MR imaging. Most of these brain neoplasms have been subjected to radiation and/or chemotherapy, and many of the tumors do not have specific imaging characteristics that will enable the neuroradiologist to discriminate tumor recurrence from the inflammatory or necrotic change that can result from treatment with radiation and/or chemotherapy. Both entities typically demonstrate contrast enhancement. It is, therefore, often the clinical course, a brain biopsy, or imaging over a lengthy follow-up interval that enables the distinction of recurrent tumor from a treatment-related lesion, not the specific imaging itself. ${ }^{3}$ A noninvasive tool that could differentiate these entities when a new enhancing lesion is first identified

From the Division of Neuroradiology, Department of Radiology, University of Michigan Health Systems, Ann Arbor, Mich. Dr. Sundgren is also affiliated with the Diagnostic Centre for Imaging and Functional Medicine, University Hospital Malmoe, University of Lund, Malmoe, Sweden

Please address correspondence to Pia C. Sundgren, MD, PhD, Professor in Radiology, Division of Neuroradiology, Department of Radiology, University of Michigan Health Systems, 1500 E Medical Center Dr, Ann Arbor, Ml 48109; e-mail: sundgren@ med.umich.edu

Indicates open access to non-subscribers at www.ajnr.org

DOI 10.3174/ajnr.A1580 would be invaluable. MR spectroscopy might be well suited for this purpose, provided that spectra of diagnostic quality can be obtained. This noninvasive imaging can be performed by using different techniques, depending on clinical question and localization of the lesion. Spectra can be acquired by using single-voxel spectroscopy (SVS) or multivoxel spectroscopy, also referred as chemical shift imaging (CSI), with both 2D CSI and, lately, 3D CSI acquisitions. Both SVS and multivoxel techniques have lately been used in the evaluation of contrastenhancing brain lesions in patients previously treated for brain neoplasms. The decision of which sequences (SVS versus 2D CSI or 3D CSI) and which parameters (ie, TE and TR) to use depends on the location of the lesion and the choice of the brain metabolites the investigator wants to evaluate.

\section{Other Radiologic and Nuclear Medicine Methods to Discriminate Radiation Injury from Recurrent or Progressive Tumor}

MR spectroscopy is not the only method used to differentiate radiation injury from recurrent tumor. Other methods that recently have been used for this purpose but that will not be discussed in detail here are positron-emission tomography (PET), diffusion-weighted imaging (DWI), MR perfusion, and CT perfusion. ${ }^{4-9}$ Some of these techniques seem to have a lower yield than others and have demonstrated lower sensitivity and specificity than MR spectroscopy, whereas some of them seem very promising.

Previous PET studies have shown that areas of radiation injury have lower glucose metabolism than normal brain tissue because they have lower cellular attenuation. ${ }^{10} \mathrm{~A}$ previous PET review reported the sensitivity of PET to be $80 \%-90 \%$ and the specificity to be $50 \%-90 \%$ in differentiating late-delayed radiation injury from recurrent high-grade glioma. ${ }^{4} \mathrm{An}$ other study of 15 patients with histopathologically confirmed diagnosis reported that fluorodeoxyglucose-PET (FDG-PET) was only $43 \%$ sensitive in distinguishing recurrent tumor from radiation effect and was the least accurate when the lesion volume was $<6 \mathrm{cc}^{11}$

Recent studies using DWI ${ }^{6}$ have shown that the apparent diffusion coefficient (ADC) ratios in the contrast-enhancing lesion are lower in recurrent tumor than in radiation-induced injury ${ }^{6}$; however, other investigators using diffusion tensor imaging (DTI $)^{7}$ have demonstrated higher ADC values in the contrast-enhancing part of the lesion in patients with tumor recurrence than in the contrast-enhancing lesion in patients with radiation injury. That study also showed that the ADC ratios in the white matter tracts in the perilesional edema were significantly higher in patients with radiation injury compared 
with those with recurrent tumor and that the fractional anisotropy (FA) ratios were significantly higher in normal-appearing white matter tracts adjacent to the edema in patients diagnosed with radiation injury compared with those with recurrent tumors. ${ }^{7}$ Thallium $201\left({ }^{201} \mathrm{Tl}\right)$ or $99 \mathrm{mTc}$ hexamethylpropyleneamine oxime single-photon emission tomography (HMPAO SPECT) or both ${ }^{12-14}$ have been reported as useful techniques to discriminate tumor progression and radiation injury. It has been suggested that the combination of lowthallium and low-HMPAO uptake was associated with benign radiation change; whereas increased uptake of either agent or both was associated with recurrent/persistent tumor at biopsy and a poor prognosis. ${ }^{14}$ However, false-positive FDG-PET and ${ }^{201} \mathrm{Tl}$ SPECT findings have been reported with biopsyproved radiation necrosis. ${ }^{15}$ In a recent CT perfusion study, the investigators demonstrated significant differences between recurrent tumor and radiation necrosis; patients with recurrent tumor had higher mean normalized cerebral blood volume (nCBV) and normalized cerebral blood flow (nCBF) and shorter normalized mean transit time compared with those with radiation necrosis. ${ }^{9}$

Newer MR imaging techniques such as dynamic susceptibility contrast (DSC) perfusion MR imaging have made it possible to obtain hemodynamic measurements such as relative $\mathrm{CBV}$, relative peak height, and percentage of signal-intensity recovery (PSR) within the brain. A recent DSC MR perfusion study of 33 patients treated with stereotactic gamma knife radiosurgery who subsequently developed progressively enlarging regions of contrast enhancement within the radiation field, suggestive of tumor recurrence or radiation necrosis, found that PSR, an imaging indicator of microvascular leakiness, was the most significant variable able to differentiate retrospectively whether a progressively enhancing lesion was due to recurrent metastatic tumor or gamma knife-induced radiation necrosis. $^{16}$

\section{Effects of Radiation on Normal Brain}

Before reviewing the role of MR spectroscopy in discriminating recurrent tumor from radiation injury, it is important to understand the underlying mechanisms resulting in radiation injury and its symptoms and also to understand what happens to the normal brain when it is irradiated.

The pathophysiology of radiation therapy-induced injury to the central nervous system (CNS) is not completely understood. Variables that might play a part include total radiation dose, size of the radiation field and radiation fraction, number and frequency of radiation doses, combination of chemotherapy and radiation therapy, duration of survival, and the age of the patient at time of treatment. ${ }^{17,18}$ For example, it is known that Adriamycin (doxorubicin) and methrotrexate potentiate radiation effects and that young children are more susceptible to radiation than adults. Radiation injury can be divided into acute, early-delayed, and late-delayed stages. ${ }^{4}$

It is believed that at least 3 different types of CNS tissue are affected by irradiation: the neurons, the glial cells, and the vessels. ${ }^{19}$ Effects on the fibrinolytic enzyme system and immune mechanisms have also been suggested. ${ }^{20,21}$ It has been shown that oligodendrocytes are very sensitive to radia$\operatorname{tion}^{21,22}$; and even if the neurons are less sensitive than the oligodendrocytes, ${ }^{19}$ there is sufficient loss of cellular compo- nents to explain the brain-volume loss seen after radiation. ${ }^{18}$ Injury to the vasculature can be seen both in early-delayed and late-delayed radiation injuries, with changes in capillary permeability, resulting in edema in the acute phase, followed by vascular endothelial damage in the chronic phase of radiation injury. A previous study has demonstrated absence of tissue plasminogen activator and excess of urokinase plasminogen activator in patients with radiation-induced necrosis, which may contribute to the cytotoxic edema and the tissue necrosis seen in the acute phase of a radiation injury. ${ }^{20}$

The incidence of radiation necrosis after conventional therapy ranges from $5 \%$ to $24 \% .^{18}$ The delayed neurologic symptoms include functional and cognitive impairments, with deficits in learning, working memory, executive function, vision, motor function, and, eventually, dementia. ${ }^{23-26} \mathrm{On}$ conventional MR imaging, the effects of radiation on brain tissue are evident in some patients as early as $2-6$ months after completion of radiation therapy as signal-intensity abnormality in white matter. ${ }^{27}$ These changes are defined as earlydelayed radiation-induced injury. Signal-intensity abnormality in the periventricular white matter has also been observed but usually not until 12-18 months after radiation therapy. ${ }^{27,28}$ Changes observed in animal models ${ }^{29-31}$ and postmortem human brain specimens ${ }^{32}$ include brain inflammation, demyelization of white matter, breakdown of the blood-brain barrier, and an array of neurotoxic effects. ${ }^{33}$ A spatial relationship between the local radiation therapy dose and the changes in the brain seen on CT or MR imaging has been noted from retrospective studies. ${ }^{27,34}$ In addition, it has been demonstrated that normal-appearing large white matter bundles such as the genu and splenium of the corpus callosum show progressive structural degradation after radiation therapy. This is evident initially in high-dose regions and later becomes evident outside the high-dose regions. ${ }^{35}$

Recent prospective studies have used MR spectroscopy to prove that structural degradation in cerebral tissue after radiation therapy can be predicted by early changes in metabolic activity before the development of neurocognitive symptoms or anatomic changes seen on conventional MR imaging. ${ }^{36,37}$ Significant alterations in brain metabolites, especially a decrease in $\mathrm{N}$-acetylaspartate (NAA), which is considered a neuronal marker, were shown to occur in normal-appearing human brain parenchyma early during radiation treatment; interval progression of these changes was noted during at least a 6-month period. ${ }^{36}$ The decrease in NAA has been suggested to be due to neuronal damage such as neuronal cell death due to apoptosis or neuronal dysfunction secondary to the irradiation. ${ }^{38}$ Other explanations such as neuronal response to blood-brain barrier breakdown, edema, damaged oligodendrocytes, demyelinization, release of cytokines, and exposure to inflammatory cells have also been suggested. ${ }^{19,39,40}$

The second important metabolite that seems to be affected by irradiation is the choline (Cho) compound, which is correlated with cell membrane biosynthesis and metabolic turnover in proliferative tissue. ${ }^{41}$ Observations of decreases in both the Cho and the Cho compounds and a decrease in the Cho/ Creatine $(\mathrm{Cr})$ ratio have been reported in irradiated brain. ${ }^{36,37,42,43} \mathrm{~A}$ third large metabolite present in the normal spectra is Cr. Cr is a marker of energy metabolism and is commonly considered to be fairly stable under most conditions 

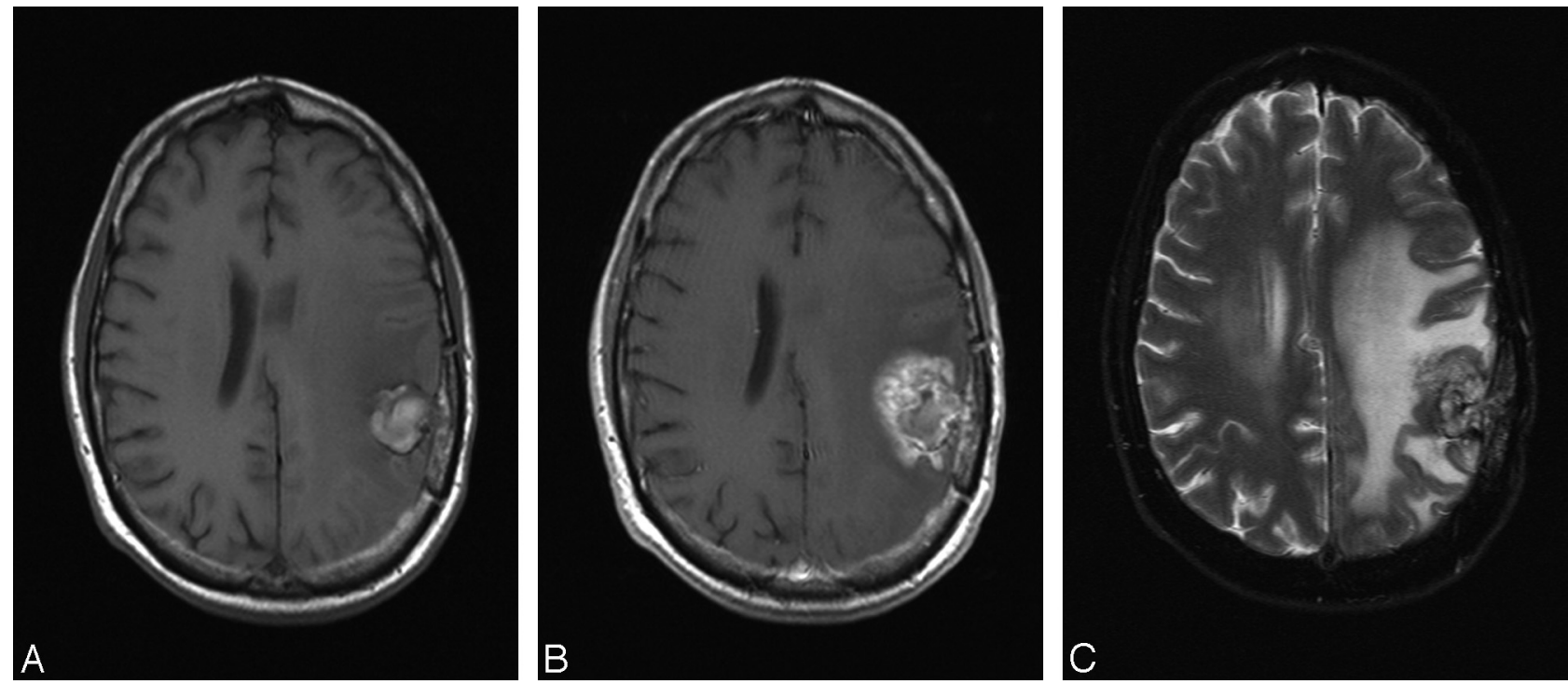

Fig 1. A, Axial noncontrast T1-weighted MR image obtained 18 months after resection and radiation of an anaplastic astrocytoma presenting with a hemorrhagic lesion in left parietal region. $B$, Postcontrast T1-weighted MR image demonstrates feathery Swiss cheese-like contrast enhancement surrounding the hemorrhagic lesion, suggestive of radiation injury. $C$, Axial T2-weighted MR image shows the extensive edema surrounding the lesion in the left hemisphere.
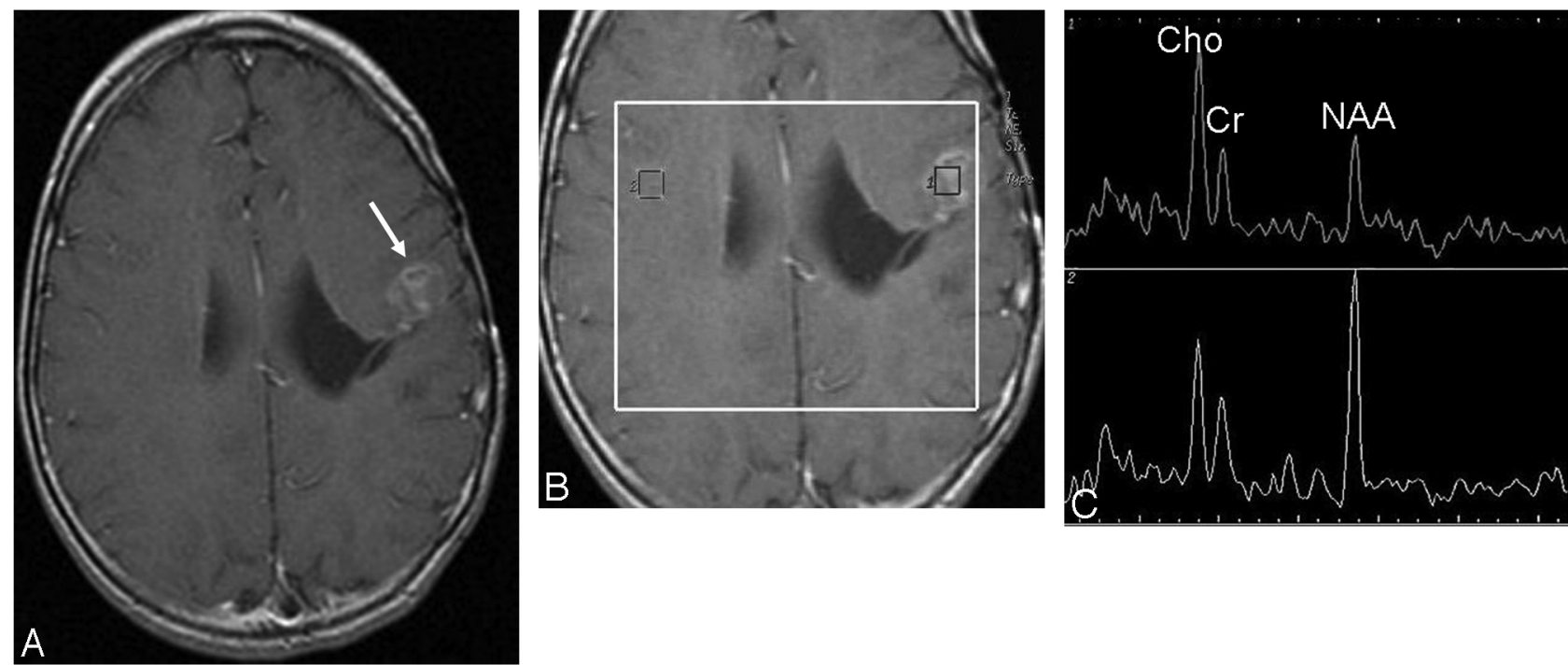

Fig 2. A, Postcontrast T1-weighted MR image obtained 12 months after resection and radiation of an ependymoma shows new contrast-enhancing lesions within the irradiated volume suspicious for tumor recurrence (arrow). B, 2D CSI MR spectroscopy (point-resolved spectroscopy sequence; TE, 144 ms, TR, 1500 ms) with manually placed voxels in the contrast-enhancing lesion and in the corresponding region in the contralateral hemisphere. $C,{ }^{1} \mathrm{H}-\mathrm{MR}$ spectrum shows moderately increased Cho and reduced NAA signal intensities (upper row), consistent with recurrent tumor, and normal signal intensities of NAA, Cho, and $\mathrm{Cr}$ in the right hemisphere (lower row).

and, therefore, is often used as the denominator in metabolic ratio calculations, even if some reports question the stability of Cr in tumors, hypoxia, and other confounding factors. ${ }^{41,44}$

Injury to the white matter outside the area of the initial brain lesion has not only been demonstrated with MR spectroscopy but is also supported by findings with DTI. For example, a recent study showed that the mean FA value decreased and the average of the mean isotropic ADC value increased significantly in normal-appearing white matter in patients treated with radiation compared with values found in normal white matter in control subjects. ${ }^{45}$

\section{MR Spectroscopy in Radiation Injury}

Conventional MR imaging alone cannot reliably discriminate tumor recurrence/progression from the inflammatory or ne- crotic changes resulting from radiation, ${ }^{3}$ though the latter can be associated with more specific patterns of enhancement, like "soap bubbles" or "Swiss cheese"17; both recurrent tumors and radiation injury typically demonstrate contrast enhancement (Fig 1). Common and uncommon features of radiationinduced injury include enhancement at the site of the original lesion and distant from the original lesion or as multiple necrotic enhancing masses spread in the brain, simulating brain metastases (Figs 2 and 3). In a recent study of 11 patients with histology-proved radiation necrosis, the most common MR imaging finding was Swiss cheese-like enhancement with feathery margins and central necrosis. ${ }^{46}$ In addition to the contrast-enhancing lesions, high T2 signal intensity in the periventricular and deep white matter with no enhancement is a common feature in patients who have been irradiated. 

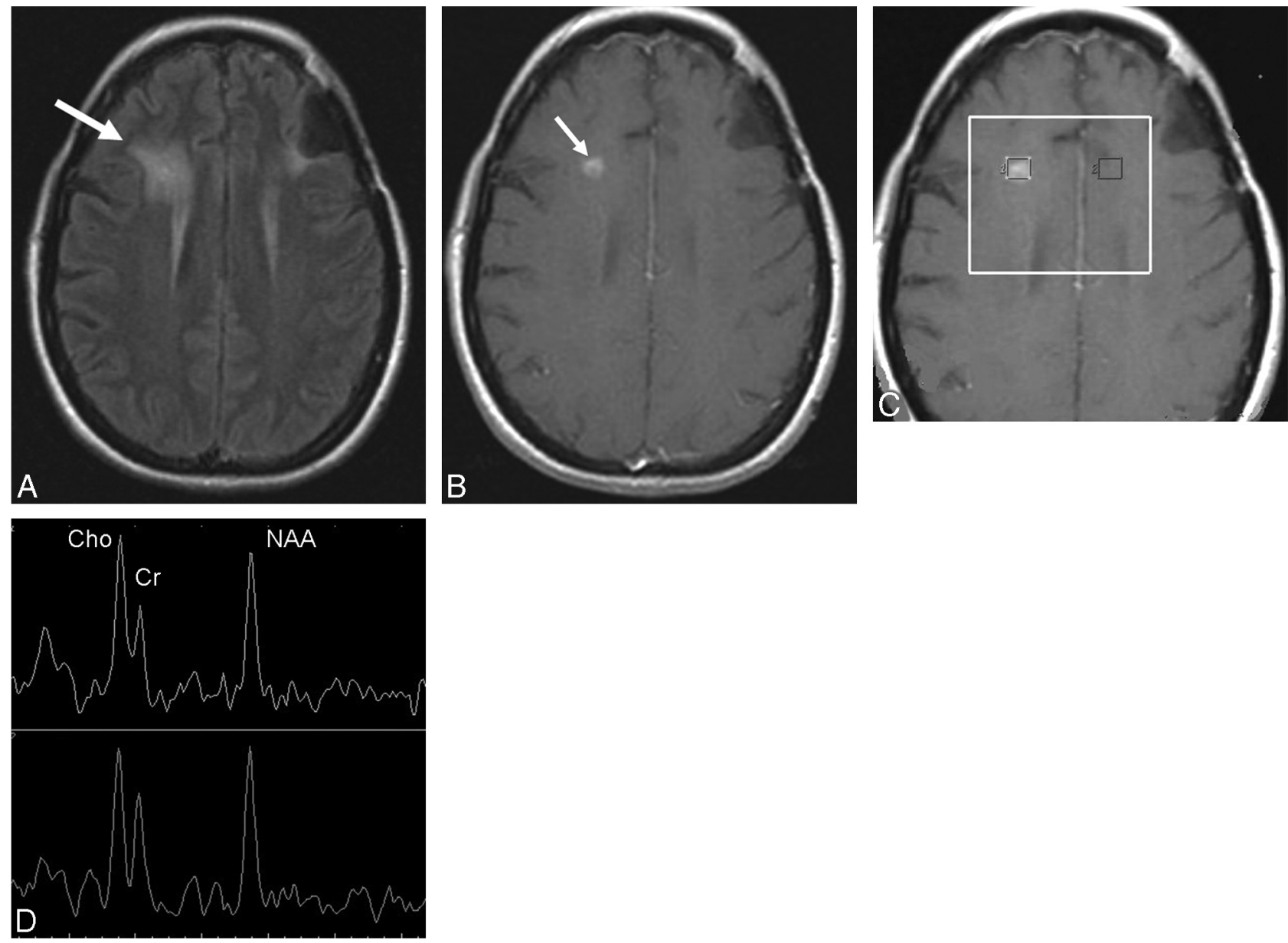

Fig 3. $A$ and $B$, Fluid-attenuated inversion recovery (FLAIR) $(A)$ and postcontrast T1-weighted MR $(B)$ images obtained 8 months after resection, radiation, and chemotherapy of an anaplastic oligodendroglioma in the left frontal lobe show a new area of hyperintensity on FLAIR (arrow, $A)$ and a contrast-enhancing nodule (arrow, B) in the right frontal lobe within the irradiated volume, suspicious for radiation injury. C, Multivoxel 2D CSI MR spectroscopy (point-resolved spectroscopy sequence; TE, $144 \mathrm{~ms}$; TR, $1500 \mathrm{~ms}$ ) with manually placed voxels in the contrast-enhancing lesion and in the corresponding area in the left hemisphere. D, MR spectroscopy spectrum shows slightly decreased NAA and increased Cho signal intensities bilaterally, suggestive of radiation injury. A follow-up MR imaging (not shown) showed interval resolution of the enhancement and no new lesions.

Specific spectroscopic changes that occur in radiation necrosis have been reported and include slight depression of NAA and variable changes in Cho and $\mathrm{Cr}^{47-50}$ In addition, radiation necrosis may show a broad peak between 0 and 2 ppm, probably reflecting cellular debris containing fatty acids, lactate (Lac), and amino acids. ${ }^{51}$ Also, other metabolites have been suggested to be present in radiation necrosis. In 1 study monitoring the progression of severe cerebral radiation injuries in the temporal lobes of 10 patients previously treated for nasopharyngeal carcinoma, an unknown resonance named Px in the 2.37- to 2.40-ppm region was detected in the affected temporal lobes in 4 of the 10 patients. ${ }^{52}$ The resonance of $\mathrm{Px}$ was only confined to spectra with Lac and in patients with the highest severity grade of radiation injury. Lesions with Px had significantly higher Lac/Cr ratios and more extensive masseffect changes than lesions without Px. The authors speculated whether Px could be associated with anaerobic glycolysis producing pyruvate (2.37 ppm) or succinate (2.40 ppm) as can be seen in brain abscess formations. ${ }^{52}$

Many of the newly occurring lesions that are subjected to MR spectroscopy do not consist only of large areas of pure tumor or radiation injury/necrosis. It has to be assumed that, more commonly, a mixture of tumor cells and tissue with radiation injury is present. This assumption is supported by a prior study of multivoxel MR spectroscopy that found that "spectral patterns do allow reliable differential diagnostic statements to be made when the tissues are composed of either pure tumor or pure necrosis, but the spectral patterns are less definitive when tissues composed of varying degrees of mixed tumor and necrosis are examined." 53

The effort to separate tumor recurrence from pure radiation damage might be more problematic when using SVS compared with using 2D CSI. SVS of an enhancing lesion that contains a small focus of recurrent tumor in a bed of much larger radiation necrosis would likely be "averaged out" such that the Cho and NAA metabolite profiles may suggest only inflammatory changes and the recurrent tumor would be missed. In addition, an enhancing lesion containing heterogeneous areas of normal CNS tissue and recurrent tumor also could be averaged into a spectral profile suggestive of only inflammatory changes, with the recurrent tumor being missed. Averaging normal or radiation-injured brain tissue with tumor tissue will tend to lower $\mathrm{Cho} / \mathrm{Cr}$ and Cho/NAA ratios of the pure tumor and lower its conspicuity, the later being a matter of major clin- 

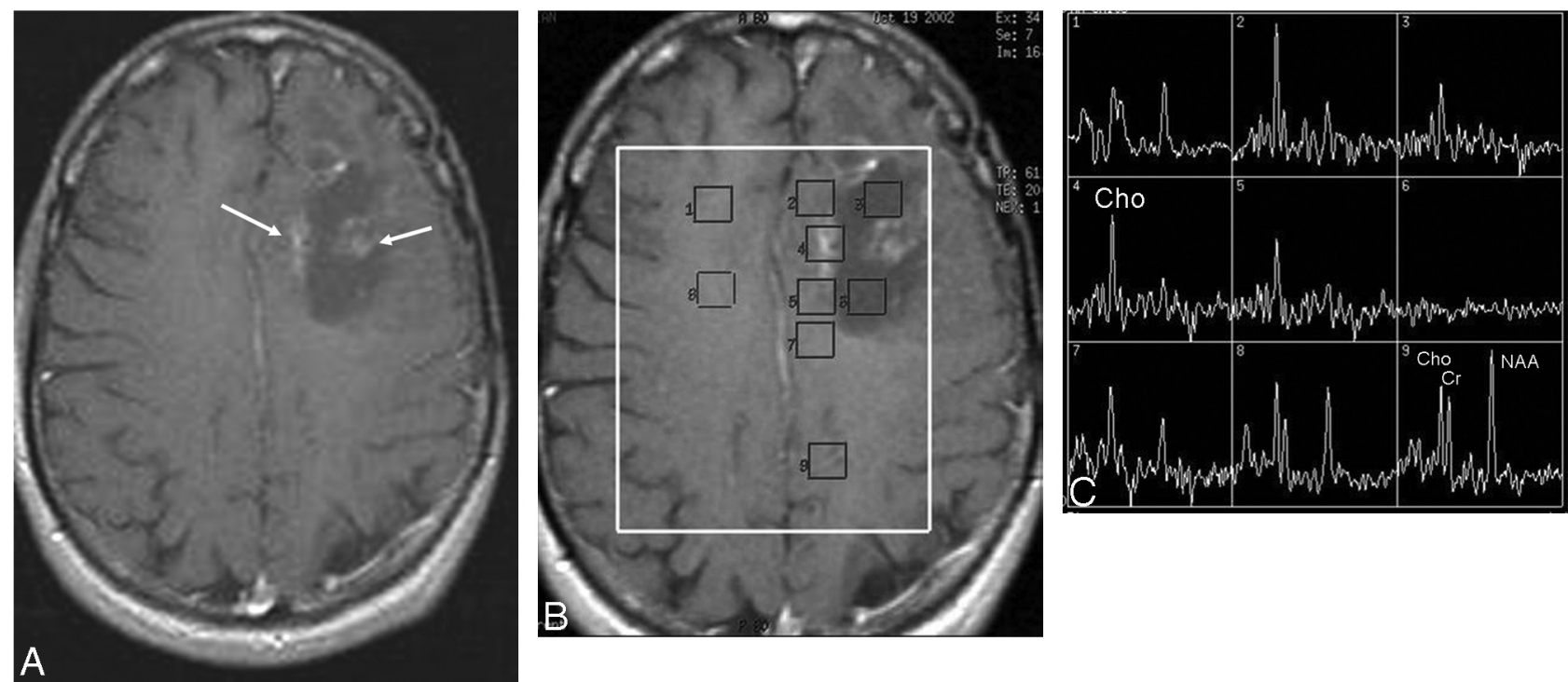

Fig 4. $A$, Postcontrast T1-weighted image obtained 12 months after resection, radiation, and chemotherapy of an astrocytoma in the left frontal lobe shows diffuse feathery contrast-enhancing areas in the vicinity of the resection cavity within the irradiated volume, suspicious for tumor recurrence. $B$, Multivoxel $2 \mathrm{D}$ CSI MR spectroscopy (point-resolved spectroscopy sequence; TE, $144 \mathrm{~ms}$; TR, $1500 \mathrm{~ms}$ ) with manually placed voxels in contrast-enhancing areas, in the cystic cavity, and in normal-appearing brain parenchyma in both left and right hemispheres. $C$, Significantly increased Cho and almost-absent NAA signal intensities in the contrast-enhancing areas, consistent with tumor recurrence verified at histopathology.

ical importance. With 2D CSI, the coverage of contrastenhancing tissue, surrounding tissue, and normal-appearing white matter in the contralateral hemisphere is allowed (Fig 4). This enables sampling of multiple discrete regions, which may be necessary to discern the subtle differences between tumor recurrence and radiation injury and for the identification of areas of both tumor and inflammatory changes in the same enhancing lesion.

A previous study using the $2 \mathrm{D}$ CSI technique reported a $97 \%$ success rate for retrospectively differentiating recurrent tumor from radiation injury, with significantly increased Cho/ $\mathrm{NAA}$ and Cho/Cr ratios in areas of recurrent tumor compared with areas of radiation injury and with normal adjacent brain tissue. ${ }^{54}$ That study reported that when cutoff values of 1.8 for either $\mathrm{Cho} / \mathrm{NAA}$ or $\mathrm{Cho} / \mathrm{Cr}$ were used (ie, values $>1.8$ being diagnostic for tumor recurrence), 27 of 28 patients were retrospectively correctly diagnosed. ${ }^{54}$ In a similar population examined with SVS, similar significant differences were found, and the use of $\mathrm{Cho} / \mathrm{Cr}$ and $\mathrm{Cho} / \mathrm{NAA}$ ratios allowed a correct retrospective classification in $>80 \%$ of the cases..$^{50}$ These findings are in agreement with those in a previous study using multivoxel ${ }^{1} \mathrm{H}$-magnetic resonance spectroscopy imaging $\left({ }^{1} \mathrm{H}-\mathrm{MRSI}\right)$ and correlation with histologic specimens. ${ }^{55} \mathrm{In}$ that study, the investigators claimed that a $\mathrm{Cho} / \mathrm{Cr}$ ratio $>1.79$ or a lipid (Lip) and Lac/Cho ratio $<0.75$ has sevenfoldincreased odds of being pure tumor compared with pure necrosis and the odds of the biopsy's being pure necrosis and having either the $\mathrm{Cho}$ /normalized $\mathrm{Cr}(\mathrm{nCr})$ values $<0.89$ or a Cho/normalized Cho (nCho) value $<0.66$ are 6 times the odds of the biopsy's being pure tumor. ${ }^{55}$

Also multivoxel 3D proton MR spectroscopy $\left({ }^{1} \mathrm{H}-\mathrm{MR}\right.$ spectroscopy) has been used in the assessment of recurrent contrast-enhancing areas at the site of the previously treated gliomas. ${ }^{56}$ In that study, the investigators found the Cho/NAA and $\mathrm{Cho/Cr}$ ratios to be significantly higher in recurrent tumor than in radiation injury, whereas the NAA/Cr ratios were lower in recurrent tumor than in radiation injury. They also noted that the $\mathrm{Cho} / \mathrm{Cr}$ and $\mathrm{Cho} / \mathrm{NAA}$ ratios were significantly higher in radiation injury than in normal-appearing white matter; however, the NAA/Cr ratios were significantly lower in radiation injury than in normal-appearing white matter. When they used receiver operating characteristic analysis, the resulting sensitivity, specificity, and diagnostic accuracy of 3D ${ }^{1} \mathrm{H}-\mathrm{MR}$ spectroscopy were $94.1 \%, 100 \%$, and $96.2 \%$, respectively, based on the cutoff values of 1.71 for $\mathrm{Cho} / \mathrm{Cr}$ or 1.71 for Cho/NAA or both as tumor criteria. ${ }^{56}$

Another ratio that has been used in the attempt to diagnose radiation necrosis is the Cho/Lip or Lac ratio. ${ }^{57}$ The authors of that study found that in cases of radiation necrosis, a high lipid-dominant peak was observed from the central nonenhanced region, along with a low Cho peak and a low NAA peak. The positive predictive value of a Cho/Lip or Lac ratio $<0.3$ and the positive predictive value of a $\mathrm{Cho} / \mathrm{Cr}<2.48$ for diagnosing radiation necrosis were $100 \%$ and $71.4 \%$, respectively. ${ }^{57}$ They concluded that it is possible to differentiate statistically radiation necrosis from metastatic brain tumor by using the $\mathrm{Cho} / \mathrm{Cr}$ ratio or the Cho/Lip or Lac ratio. However, they found no significant difference between glioblastoma and radiation necrosis by using the $\mathrm{Cho} / \mathrm{Cr}$ ratio. ${ }^{57}$

Similar figures were seen in another study by the same group in which the authors could differentiate ring-enhancing lesions as "space-occupying radiation necrosis" from ring-enhancing metastasis in all 6 cases by using MR spectroscopy. ${ }^{58}$

With recurrent/residual tumor, the pathologic spectra consistent with the presence of tumor (ie, markedly elevated Cho and depressed NAA) can be identified not only in voxels placed within the contrast-enhancing lesion but also in voxels outside the contrast-enhancing lesion, as demonstrated in $38 \%$ of the cases in a recent study. ${ }^{54}$

It has been suggested that the radiation dosage plays a role in the changes in metabolic ratios. An increase in Cho/ $\mathrm{Cr}$ ratios after radiation therapy, proportional to radiation dose, has been reported from studies using SVS ${ }^{43,50,51}$; a decrease in NAA or a reduction in NAA/Cr ratios or both af- 

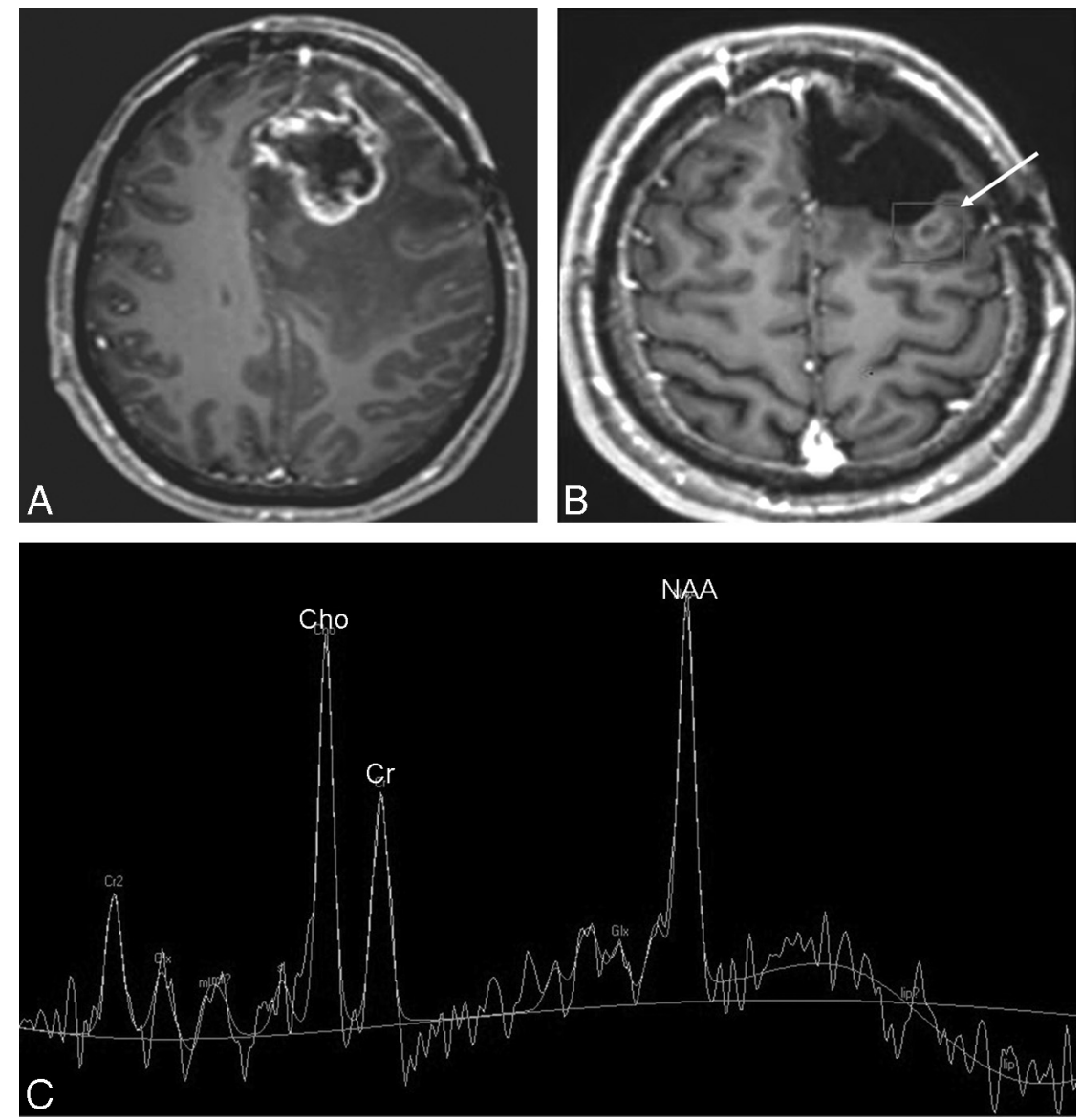

Fig 5. A, Postcontrast T1-weighted image obtained 10 months after resection and radiation of an astrocytoma in the left frontal lobe shows an irregular peripherally contrast-enhancing mass lesion with central necrosis surrounded by edema suspicious for tumor recurrence. The patient had the lesion resected, and histopathology revealed a high-grade astrocytoma. $B$ At follow-up MR imaging 6 months later and after additional radiation, a new diffuse contrast-enhancing lesion was present within the irradiated volume. ${ }^{1} \mathrm{H}$-MR spectroscopy by using SVS (point-resolved spectroscopy sequence; TE, 144 ms; TR, 2000 ms) was performed with the volume placed in over the contrast-enhancing lesion. C, Slightly increased Cho and normal NAA and $\mathrm{Cr}$ signal intensities are indicative of radiation injury, which was histopathologically confirmed after additional resection.

ter radiation therapy are also a frequently reported finding (Fig 5). ${ }^{41-43,47,48,50,51,59}$

\section{Measurements of Metabolites and Ratio Calculations}

One of the major problems when reviewing the literature and comparing existing MR spectroscopy data is the use of various ways of calculating metabolite ratios. Several different methods have been used when trying to differentiate recurrent tumor from radiation-induced injury, and there is currently no consensus in the literature on which type of differently calculated ratios can best differentiate tumor recurrence from radiation change. Standard metabolite ratios calculated from data obtained within the diagnostic voxel (eg, Cho/NAA) have been used in many institutions ${ }^{54,60-64}$ and sometimes compared with similar ratios obtained from the contralateral hemisphere. ${ }^{65}$ Alternatively, normalized ratios have been calculated by using a variety of definitions such as the following: 1) using 1 metabolite from the diagnostic voxel in the numerator and the other (or same) metabolite from the contralateral hemisphere in the denominator (eg, Cho/normalized NAA [nNAA]), ${ }^{53,55,63,66}$ 2) dividing a metabolite peak area measured in the lesion by the sum of all the spectrum peak areas in the same lesion, ${ }^{41}$ and 3) dividing a metabolite peak integral in the lesion by the same metabolite peak in the "normal brain" and comparing it with standard metabolite ratios. ${ }^{67}$
Other more tumor-specific ways of spectrum evaluation, like the Cho-NAA index, have been used to assess the presence of tumor recurrence. ${ }^{68}$ In a recent study, the 2 most common ways of calculating ratios were compared: Normalized ratios in which the metabolite from the normal contralateral hemisphere was used as denominator were compared with "nonnormalized" ratios obtained from metabolites in the lesion (both as the numerator and the denominator). ${ }^{69}$ That study demonstrated that 2 non-normalized ratios, Cho/NAA and $\mathrm{NAA} / \mathrm{Cr}$, were significantly correlated with tumor recurrence, whereas only 1 of the 6 normalized ratios tested, Cho/nNAA, significantly correlated with tumor recurrence. Non-normalized Cho/NAA was the best discriminator for predicting tumor recurrence, with a sensitivity of $86 \%$, a specificity of $90 \%$, a positive predictive value of $92 \%$, a negative predictive value of $81 \%$, and $88 \%$ correctly classified. Non-normalized Cho/ NAA performed the best (area under the receiver operating characteristic analysis [ROC] curve $=0.92$ ), followed by $\mathrm{NAA} / \mathrm{Cr}$ (area under the ROC curve $=0.85$ ). Cho/nNAA was the only ratio to yield significant values for tumor recurrence with a sensitivity of $73 \%$, specificity of $40 \%$, a positive predictive value of $64 \%$, a negative predictive value of $50 \%$, and $60 \%$ correctly classified; the area under the ROC curve was $0.77 .^{69}$

One major drawback of most of the studies in this field, regardless of the ways of calculating the metabolite ratios, is 
the limited correlation with histopathology. From 1 of the few larger reports with correlating histopathology, it is clear that MR spectroscopy is a helpful tool in differentiating pure necrosis from normal tissue and from pure tumor; however, MR spectroscopy has problems in differentiating when the specimen is a mixture of recurrent tumor and radiation necrosis. ${ }^{55}$ That study also found that combining the information from ADC values with the MR spectroscopy ratios did not help much when trying to distinguish specimens of mixed tumor and the addition of ADC did not increase the accuracy in identifying pure tumor or pure necrosis compared with MR spectroscopy alone. ${ }^{56}$

\section{Need for Prediction Models for Clinical Decision Making}

Although previous studies have shown that MR spectroscopy findings affect the clinical management of patients with brain tumor, ${ }^{62,70}$ the question remains whether MR spectroscopy alone or combined with other methods can affect clinical management of patients with new contrast-enhancing lesions after radiation.

Although specific ratio cutoffs may be helpful in defining the ability of the MR spectroscopy to detect statistically significant differences between patients with recurrent tumor and those with radiation injury/necrosis, a more clinically applicable measure of the utility of a test, in this case MR spectroscopy, is needed. The clinical relevance of a positive or negative test may be estimated by the corresponding likelihood ratio. ${ }^{71}$ Although likelihood ratios are effective tools in guiding selection of a diagnostic test, clinical decision making at the level of the individual patient relies ultimately on the assignment of posttest probability - that is, the probability of disease, given the test result. Posttest probabilities of recurrent tumor can be explicitly quantified for the individual patient by using a prediction model. Recently such a model has been proposed, ${ }^{72}$ which uses alterations in the ratios of standard brain metabolites to predict the probability of tumor recurrence in patients previously treated for brain tumors with new contrastenhancing lesions. ${ }^{72}$ Because the model predicts the probability of tumor recurrence, it allows classification of patients into different clinical management strategies by using ranges of posttest probability, which may facilitate clinical decision making.

In the future, prediction models combining multiple metabolic ratios, with or without clinical data, may prove to be an even more effective decision-making tool, resulting in reduction of the number of patients subjected to unnecessary invasive procedures or treatment.

In the future, not only are more sophisticated prediction models needed but also larger multicenter studies validating such models and larger prospective studies using new methods like 3D MR spectroscopy and target-guided biopsies with histologic confirmation to reach a consensus about the true value of MR spectroscopy as a method of differentiating recurrent tumor from radiation injury. Future investigations should be focused on trying to determine if MR spectroscopy alone can be the tool that is needed or if the technique should be used only in combination with other radiologic methods to obtain the highest sensitivity, specificity, and accuracy.

\section{Conclusions}

MR spectroscopy is presently one of the noninvasive radiologic methods used to distinguish recurrent tumor and radiation injury in patients previously treated with radiation for brain neoplasm. Still, despite a considerable volume of research in the field, no consensus exists in the community regarding ratio calculations, the accuracy of MR spectroscopy to identify radiation necrosis, and the accuracy of MR spectroscopy in differentiating radiation necrosis from tumor recurrence or the true value of the method in clinical decision making.

\section{Acknowledgment}

I thank Eva Prahl for her editing, formatting, and assistance in the preparation of this manuscript.

\section{References}

1. Walker MD, Strike TA, Sheline GE. An analysis of dose-effect relationship in the radiotherapy of malignant gliomas. Int J Radiat Oncol Biol Phys 1979;5:1725-31

2. Johannesen TB, Lien $\mathrm{HH}$, Hole $\mathrm{KH}$, et al. Radiological and clinical assessment of long-term brain tumour survivors after radiotherapy. Radiother Oncol 2003;69:169-76

3. Bonavita S, Di Salle F, Tedeschi G. Proton MRS in neurological disorders. Eur J Radiol 1999;30:125-31

4. Langleben DD, Segall GM. PET in differentiation of recurrent brain tumor from radiation injury. J Nucl Med 2000;41:1861-67

5. Terakawa Y, Tsuyuguchi N, Iwai Y, et al. Diagnostic accuracy of 11C-methionine PET for differentiation of recurrent brain tumors from radiation necrosis after radiotherapy. J Nucl Med 2008;49:694-99

6. Hein PA, Eskey CJ, Dunn JF, et al. Diffusion-weighted imaging in the follow-up of treated high-grade gliomas: tumor recurrence versus radiation injury. AJNR Am J Neuroradiol 2004;25:201-09

7. Sundgren PC, Fan X, Weybright P, et al. Differentiation of recurrent brain tumor versus radiation injury using diffusion tensor imaging in patients with new contrast enhancing lesions. Magn Reson Imaging 2006;24:1131-42. Epub 2006 Sep 18

8. Sugahara T, Korogi Y, Tomiguchi S, et al. Posttherapeutic intraaxial brain tumor: the value of perfusion-sensitive contrast-enhanced MR imaging for differentiating tumor recurrence from nonneoplastic contrast-enhancing tissue. AJNR Am J Neuroradiol 2000;21:901-09

9. Jain R, Scarpace L, Ellika S, et al. First-pass perfusion computed tomography: initial experience in differentiating recurrent brain tumors from radiation effects and radiation necrosis. Neurosurgery 2007;61:778-86

10. Di Chiro G, Oldfield E, Wright DC. Cerebral necrosis after radiotherapy and/or intraarterial chemotherapy for brain tumors: PET and neuropathologic studies. AJR Am J Roentgenol 1988;150:189-97

11. Thompson TP, Lunsford LD, Kondziolka D. Distinguishing recurrent tumor and radiation necrosis with positron emission tomography versus stereotactic biopsy. Stereotact Funct Neurosurg 1999;73:9-14

12. Carvalho PA, Schwartz RB, Alexander E, et al. Detection of recurrent gliomas with qualitative thallium-201/technetium 99m HMPAO single-photon emission computerized tomography. J Neurosurg 1992;77:565-70

13. Schwartz RB, Carvalho PA, Alexander E, et al. Radiation necrosis vs high-grade recurrent glioma: differentiation by using dualisotope SPECT with 201Tl and 99mTc-HMPAO. AJNR Am J Neuroradiol 1991;12:1187-92

14. Schwartz RB, Holman BL, Polak JF, et al. Dual-isotope single-photon emission computerized tomography scanning in patients with glioblastoma multiforme: association with patient survival and histopathological characteristics of tumor after high-dose radiotherapy. J Neurosurg 1998;89:60-68

15. Matheja P, Rickert C, Weckesser M, et al. Scintigraphic pitfall: delayed radionecrosis-case illustration. J Neurosurg 2000;92:732

16. Barajas RF, Chang JS, Sneed PK, et al. Distinguishing recurrent intra-axia metastatic tumor from radiation necrosis following gamma knife radiosurgery using dynamic susceptibility-weighted contrast-enhanced perfusion MR imaging. AJNR Am J Neuroradiol 2009;30:367-72. Epub 2008 Nov 20

17. Kumar AJ, Leeds NE, Fuller GN, et al. Malignant gliomas: MR imaging spectrum of radiation therapy- and chemotherapy-induced necrosis of the brain after treatment. Radiology 2000;217:377-84

18. Marks JE, Baglan RJ, Prassad SC, et al. Cerebral radionecrosis: incidence and risk in relation to dose, time, fractionation and volume. Int J Radiat Oncol Biol Phys 1981;7:243-52

19. Belka C, Budach W, Kortmann RD, et al. Radiation-induced CNS toxicity: molecular and cellular mechanisms. Br J Cancer 2001;85:1233-9 
20. Sawaya R. The fibrinolytic enzymes in the biology of brain tumors. In: Swaya MD, ed. Fibrinolysis and the Central Nervous System. Philadelphia: Hanley and Belfus; 1990:106-26

21. Castel JC, Caille JM. Imaging of irradiated brain tumors: value of magnetic resonance imaging. J Neuroradiol 1989;16:81-132

22. Burger PC, Boyko OB. The pathology of central nervous system radiation injury. In: Gutin PH, Leibel SA, Sheline GE, eds. Radiation Injury to the Nervous System. New York: Raven; 1991:191-208

23. Khong PL, Leung LH, Fung AS, et al. White matter anisotropy in post-treatment childhood cancer survivors: preliminary evidence of association with neurocognitive function. J Clin Oncol 2006;24:884-90

24. Moretti R, Torre P, Antonello RM, et al. Neuropsychological evaluation of late-onset post-radiotherapy encephalopathy: a comparison with vascular dementia. J Neurol Sci 2005;229-230:195-200. Epub 2004 Dec 19

25. Cole PD, Kamen BA. Delayed neurotoxicity associated with therapy for children with acute lymphoblastic leukemia. Ment Retard Dev Disabil Res Rev 2006; $12: 174-83$

26. Herman MA, Tremont-Lukats I, Meyers CA, et al. Neurocognitive and functional assessment of patients with brain metastases: a pilot study. Am J Clin Oncol 2003;26:273-79

27. Constine LS, Konski A, Ekholm S, et al. Adverse effects of brain irradiation correlated with MR and CT imaging. Int J Radiat Oncol Biol Phys 1988;15:319-30

28. Packer RJ, Zimmerman RA, Bilaniuk LT. Magnetic resonance imaging in the evaluation of treatment-related central nervous system damage. Cancer 1986;58:635-40

29. Price RE, Langford LA, Jackson EF, et al. Radiation-induced morphologic changes in the rhesus monkey (Macaca mulatta) brain. J Med Primatol 2001;30:81-87

30. Benczik J, Tenhunen M, Snellman M, et al. Late radiation effects in the dog brain: correlation of MRI and histological changes. Radiother Oncol 2002;63:107-20

31. Chiang CS, McBride WH, Withers HR. Myelin-associated changes in mouse brain following irradiation. Radiother Oncol 1993;27:229-36

32. Burger PC, Mahley MS Jr, Dudka L, et al. The morphologic effects of radiation administered therapeutically for intracranial gliomas: a postmortem study of 25 cases. Cancer 1979;44:1256-72

33. Armstrong CL, Gyato K, Awadalla AW, et al. A critical review of the clinical effects of therapeutic irradiation damage to the brain: the roots of controversy. Neuropsychol Rev 2004;14:65-86

34. Mikhael MA. Radiation necrosis of the brain: correlation between patterns on CT and dose of radiation. J Comput Assist Tomogr 1979;3:241-49

35. Nagesh V, Tsien CI, Chenevert TL, et al. Radiation-induced changes in normalappearing white matter in patients with cerebral tumors: a diffusion tensor imaging study. Int J Radiat Oncol Biol Phys 2008;70:1002-10

36. Sundgren PC, Nagesh V, Elias A, et al. Metabolic alterations: a biomarker for radiation-induced injury of normal brain—a spectroscopy study. J Magn Reson Imaging 2009;29:291-97

37. Lee MC, Pirzkall A, McKnight TR, et al. 1H-MRS of radiation effects in normalappearing white matter: dose-dependence and impact on automated spectral classification. J Magn Reson Imaging 2004;19:379-88

38. Tofilon PJ, Fike JR. The radioresponse of the central nervous system: a dynamic process. Radiat Res 2000;153:357-70

39. Bates TE, Strangeward M, Keelan J, et al. Inhibition of $\mathrm{N}$-acetylaspartate production: implications for 1H MRS studies in vivo. Neuroreport 1992;7: $1397-400$

40. de Stefano N, Matthews PM, Arnold DL. Reversible decreases in N-acetylaspartate after acute brain injury. Magn Reson Med 1995;34:721-27

41. Estève F, Rubin C, Grand S, et al. Transient metabolic changes observed with proton MR spectroscopy in normal human brain after radiation therapy. Int $J$ Radiat Oncol Biol Phys 1998;40:279-86

42. Chan YL, Roebuck DJ, Yuen MP, et al. Long-term cerebral metabolite changes on proton magnetic resonance spectroscopy in patients cured of acute lymphoblastic leukemia with previous intrathecal methotrexate and cranial irradiation prophylaxis. Int J Radiat Oncol Biol Phys 2001;50:759-63

43. Isobe T, Matsumura A, Anno I, et al. Changes in $1 \mathrm{H}-\mathrm{MRS}$ in glioma patients before and after irradiation: the significance of quantitative analysis of choline-containing compounds [in Japanese]. No Shinkei Geka 2003;31:167-72

44. Walecki J, Sokól M, Pieniazek P, et al. Role of short TE 1H-MR spectroscopy in monitoring of post-operation irradiated patients. Eur J Radiol 1999;30: $154-61$

45. Kitahara S, Nakasu S, Murata K, et al. Evaluation of treatment-induced cerebral white matter injury by using diffusion-tensor MR imaging: initial experience. AJNR Am J Neuroradiol. 2005;26:2200-06

46. Sundgren PC, Rogers L, Tsien CT, et al. Correlation of Magnetic Resonance Imaging Morphologic Abnormalities, Magnetic Resonance Spectroscopy and Radiation Treatment Dose-Volumes in Histological Proven Cerebral Radiation Necrosis. Proceedings of the European Society of Neuroradiology, Krakow, Poland, September $18-21,2008$

47. Chong VF, Rumpel H, Fan YF, et al. Temporal lobe changes following radia- tion therapy: imaging and proton MR spectroscopic findings. Eur Radio 2001;11:317-24

48. Chong VF, Rumpel H, Aw YS, et al. Temporal lobe necrosis following radiation therapy for nasopharyngeal carcinoma: $1 \mathrm{H}$ MR spectroscopic findings. Int $J$ Radiat Oncol Biol Phys 1999;45:699-705

49. Schlemmer JP, Bachert $\mathrm{P}$, Henze M, et al. Differentiation of radiation necrosis from tumor progression using proton magnetic resonance spectroscopy. Neuroradiology 2002;44:216-22

50. Schlemmer HP, Bachert P, Herfarth K, et al. Proton MR spectroscopic evaluation of suspicious brain lesions after stereotactic radiotherapy. AJNR Am J Neuroradiol 2001;22:1316-24

51. Castillo M, Kwock L, Mukherji SK. Clinical applications of proton MR spectroscopy. AJNR Am J Neuroradiol 1996;17:1-15

52. Yeung DK, Chan $Y$, Leung $S$, et al. Detection of an intense resonance at $2.4 \mathrm{ppm}$ in $1 \mathrm{H}$ MR spectra of patients with severe late-delayed, radiation-induced brain injuries. Magn Reson Med 2001;45:994-1000

53. Rock JP, Scarpace L, Hearshen D, et al. Associations among magnetic resonance spectroscopy, apparent diffusion coefficients and image-guided histopathology with special attention to radiation necrosis. Neurosurgery 2004:54;1111-19

54. Weybright P, Sundgren PC, Maly P, et al. Differentiation between brain tumour recurrence and radiation injury using MR spectroscopy. $A J R A m J$ Roentgenol 2005;185:1471-76

55. Rock JP, Hearshen D, Scarpace L, et al. Correlations between magnetic resonance spectroscopy and image-guided histopathology, with special attention to radiation necrosis. Neurosurgery 2002;51:912-19

56. Zeng QS, Li CF, Zhang K, et al. Multivoxel 3D proton MR spectroscopy in the distinction of recurrent glioma from radiation injury. J Neurooncol 2007;84: 63-69

57. Kimura $\mathrm{T}$, Sako $\mathrm{K}$, Gotoh $\mathrm{T}$, et al. In vivo single voxel proton $\mathrm{MR}$ spectroscopy in brain lesions with ring-like enhancement. NMR Biomed 2001;14:339-49

58. Kimura T, Sako K, Tohyama Y, et al. Diagnosis and treatment of progressive space-occupying radiation necrosis following radiosurgery for brain metastasis: value of proton magnetic resonance spectroscopy. Acta Neurochir 2003; 145:557-64

59. Rutkowski T, Tarnawski R, Sokol M, et al. Proton-MR spectroscopy of norma brain tissue before and after postoperative radiotherapy because of primary brain tumors. Int J Radiat Oncol Biol Phys 2003;56:1381-89

60. Weybright P, Maly P, Gomez Hassan D, et al. MR spectroscopy in the evaluation of recurrent contrast enhancing lesions in the posterior fossa after tumor treatment. Neuroradiology 2004;46:541-59

61. Bulakbasi N, Kocaoglu M, Ors F, et al. Combination of single-voxel proton MR spectroscopy and apparent diffusion coefficient calculation in the evaluation of common brain tumors. AJNR Am J Neuroradiol 2003;24:225-33

62. Lin A, Bluml S, Mamelak AN. Efficacy of proton magnetic resonance spectroscopy in clinical decision making for patients with suspected malignant brain tumors. J Neurooncol 1999;45:69-81

63. Yang D, Korogi Y, Sugahara T, et al. Cerebral gliomas: prospective comparison of multivoxel $2 \mathrm{D}$ chemical-shift imaging proton MR spectroscopy, echoplanar perfusion and diffusion-weighted MRI. Neuroradiology 2002; 44:656-66

64. Wald LL, Nelson SJ, Day MR, et al. Serial proton magnetic resonance spectroscopy imaging of glioblastoma multiforme after brachytherapy. J Neurosurg 1997;87:525-34

65. Kinoshita K, Tada E, Matsumoto K, et al. Proton MR spectroscopy of delayed cerebral radiation in monkeys and humans after brachytherapy. AJNR Am J Neuroradiol 1997;18:1753-61

66. Croteau D, Scarpace L, Hearshen D, et al. Correlation between magnetic resonance spectroscopy imaging and image-guided biopsies: semiquantitative and quantitative histopathological analyses of patients with untreated glioma. Neurosurgery 2001;49:823-29

67. Lazareff JA, Gupta RK, Alger J. Variation of post-treatment H-MRSI choline signal intensity in pediatric gliomas. J Neurooncol 1999;41:291-98

68. McKnight TR, von dem Busche MH, Vigneron DB, et al. Histopathologica validation of a three-dimensional magnetic resonance spectroscopy index as a predictor of tumor presence. J Neurosurg 2002;97:794-802

69. Elias A, Carlos RC, Smith E, et al. MR Spectroscopy Using Normalized and NonNormalized Metabolite Ratios for Differentiating Recurrent Brain Tumor from Radiation Injury. Proceedings of the Radiological Society of North America, Chicago, Ill, November 30-December 5, 2008

70. Lin A, Mamelak A, Ross BD. Effect of MRS on Clinical Decision Making in Brain Tumour Management. Proceedings of the International Society of Magnetic Resonance in Medicine, Denver, Colo, April 1-7, 2000

71. Guyatt GH, Haynes RB, Jaeschke RZ, et al. Users' Guides to Medical Literature XXV: evidence-based medicine - principles for applying the Users' Guides to patient care. Evidence-Based Medicine Working Group. JAMA 2000;284 1290-96

72. Smith EA, Carlos RC, Junck LR, et al. Developing a clinical decision model: use of MR spectroscopy to differentiate between recurrent tumor and radiation change in patients with new contrast enhancing lesions. AJR Am J Roentgenol 2009; 192:W45-52 